\title{
Atemtherapie mit Ez-PAP zur Behandlung der dynamischen Überblähung bei Patienten mit schwerer COPD und Lungenemphysem
}

\author{
Respiratory Therapy with Ez-PAP for Treatment of Dynamic Hyperinflation \\ in Patients with Severe COPD and Emphysema
}

Autoren

Institute
G. Iberl' ${ }^{1}$, F. Bornitz ${ }^{1}$, M. Schellenberg ${ }^{1}$, M. Wiebel' ${ }^{1}$, F. J.F. Herth ${ }^{1,2}$, M. Kreuter ${ }^{1,2}$

${ }^{1}$ Pneumologie und Beatmungsmedizin, Thoraxklinik, Universitätsklinikum Heidelberg

${ }^{2}$ Zentrum für Translationale Lungenforschung Heidelberg (TLRC), Mitglied des Deutschen Zentrums

für Lungenforschung (DZL) eingereicht 22.2 .2014 akzeptiert nach Revision 20.6.2014

Bibliografie

Dol http://dx.doi.org/ 10.1055/s-0034-1377483 Pneumologie 2014; 68: 604-612 (c) Georg Thieme Verlag KG Stuttgart · New York ISSN 0934-8387

Korrespondenzadresse Gabriele Iberl

Atmungstherapeutin DGP Pneumologie und Beatmungsmedizin Thoraxklinik, Universitätsklinikum Heidelberg Amalienstr. 5 69126 Heidelberg gabriele.iber|@med.uniheidelberg.de

\section{Zusammenfassung \\ V}

Hintergrund: Die nichtmedikamentöse physiotherapeutische Atemtherapie gewinnt bei der schweren chronisch obstruktiven Lungenerkrankung und Lungenemphysem (COPD) zunehmend Relevanz. Ez-PAP, ein Compact-CPAP bzw. FlowPEP-System, leistet neben dem PEP-Effekt in der Exspiration Inspirationshilfe durch den CoandaEffekt.

Methode und Patienten: 30 Patienten mit schwerer COPD und Lungenemphysem mit hyperkapnischer ventilatorischer Insuffizienz wurden unter nicht- (NIV) $(\mathrm{n}=28)$ und invasiver Beatmung ( $\mathrm{n}=$ 2 ), im beatmungsfreien Intervall mit Ez-PAP behandelt und retrospektiv analysiert. Ausgewertet wurden - soweit vorliegend - klinische Verläufe wie Beatmungszeit und -abhängigkeit, Angaben zur Dyspnoe (visuelle CR-10-BORG-Skala) Ergebnisse des 6-Minutengehtests (6-MWT), lungenfunktionelle Daten und Aufzeichungen transkutaner $\mathrm{CO}_{2}$-Messungen $(\mathrm{tcpCO})_{2}$ ).

Ergebnisse: Es wurde eine Verlängerung der beatmungsfreien Zeiten bei beatmungsabhängigen Patienten $(n=9)$ erreicht, ein medianer Abfall des Dyspnoeempfindens um 3,3 Punkte $(n=10)$ und ein medianer Anstieg der Belastbarkeit um 50,4 m im 6MWT $(n=5)$. Lungenfunktionell fand sich im Median eine Steigerung der Vitalkapazität um $544 \mathrm{ml}(\mathrm{n}=5)$. Die Kombination mit manueller Entblähungstechnik führte zu einem Abfall des tcpCO $\mathrm{Cm}_{2}$ u, $3 \mathrm{mmHg}(\mathrm{n}=6)$.

Schlussfolgerung: Die Therapie mit Ez-PAP scheint die dynamische Überblähung bei der schweren COPD zu reduzieren, die Atmung zu ökonomisieren und die Atempumpe teilweise zu entlasten. Prospektive Untersuchungen dieser vielversprechenden Methode sind dringend erforderlich.

\section{Abstract \\ $\nabla$}

Background: Non-pharmacological respiratory physiotherapy in treatment of COPD with severe emphysema is achieving increasing importance. Ez-PAP, a compact CPAP- or flow-PEP system, supports inspiration by using the Coanda effect in addition to a PE(E)P-effect during expiration.

Methods and Patients: 30 patients with severe COPD and emphysema and hypercapnic respiratory failure under non-(NIV) $(\mathrm{n}=28)$ and invasive ventilation $(n=2)$ were treated in ventilator-free intervals with Ez-PAP and analyzed retrospectively. Clinical courses such as ventilator-dependency, dyspnea by visual CR-10 Borg scale, results of sixminute walk tests (6MWT), lung function tests and recordings of transcutaneous $\mathrm{CO} 2$ measurements were evaluated where possible.

Results: Analyzed patients showed less ventilator dependancy $(n=9)$, reported a median decrease of shortness of breath by 3,3 points $(n=10)$ and improved by $50,4 \mathrm{~m}$ in the 6MWT $(\mathrm{n}=5)$. A median increase of vital capacity by $544 \mathrm{ml}(\mathrm{n}=5)$ was shown by spirometry. Combined with manual techniques for reducing air-trapping, treatment success was documented by a median reduction of pCO2 by $7,3 \mathrm{mmHg}(\mathrm{n}=6)$ using transcutaneous pCO2 measurement.

Conclusion: Ez-PAP is a simple mechanical method to reduce dynamic hyperinflation in severe COPD, thus achieving relief of respiratory muscles and more effective breathing. Prospective studies of this promising method are urgently required. 


\section{Einleitung}

\section{$\nabla$}

Dyspnoe ist eines der Leitsymptome der schweren chronisch obstruktiven Lungenerkrankung (COPD), welches die Aktivitäten der Betroffenen deutlich einschränkt. Teilweise bereits in Ruhe, aber vorwiegend unter zumeist schon geringer Belastung, kommt es bei der schweren COPD mit Emphysem durch die gesteigerte Atemfrequenz, den dabei oft aggravierten exspiratorischen Atemwegskollaps und eine zunehmende Obstruktion zur dynamischen Überblähung [1,2]. Zusammen mit dem Verlust von Gasaustauschfläche, einer unvollständigen Exspiration mit Anstieg des intrinsischen positiven endexspiratorischen Drucks (iPEEP) und einem Zwerchfelltiefstand mit eingeschränkter Inspirationskapazität führen diese Veränderungen zu zunehmender Dyspnoe und Hypoxie sowie im Endstadium der Krankheit zum Atempumpenversagen mit konsekutiver Hyperkapnie [3]. Diese schwerstkranken Patienten stehen unter einem großen Leidensdruck, da die progrediente Dyspnoe nicht immer ausreichend zu behandeln ist. Zudem kommen andere, zumeist invasive Interventionen wie die endoskopische Lungenvolumenreduktion und auch die Lungentransplantation nur für relativ wenige, sorgfältig evaluierte Patienten in Frage [4,5].

Große randomisierte kontrollierte Studien zum Stellenwert der Atemphysiotherapie in der Behandlung der COPD fehlen bislang [6]. Bei den vorliegenden Studien wurden meist unterschiedliche Interventionen wie z.B. konventionelle Atemphysiotherapie, Brustkorbmobilisation, intrapulmonale Perkussion, Therapie mit PEP-Geräten etc. bei heterogenen Patientenkollektiven durchgeführt, die vor allem der Beurteilung des Stellenwerts der Sekretelimination dienen [7]. Allerdings ist der Einsatz der Lippenbremse und von mechanischen Hilfsmitteln mit exspiratorisch wirksamen Stenosen (PEP-positive expiratory pressure) zur Atemkontrolle und Vermeidung von Atemnot seit langem klinisch etabliert [8]. Als Wirkmechanismus wird einerseits die Vermeidung des exspiratorischen Kollapses durch eine intrabronchiale Druckerhöhung entsprechend einer Verschiebung des Equal Pressure Points (EPP) nach proximal [9], andererseits eine verbesserte Entlüftung über die kollaterale Ventilation diskutiert, die auch zu einer verbesserten Sekretelimination führt [10,11]. Ez-PAP ${ }^{\circledR}$, ein Flow-PEP-Gerät, wurde als Continuous Positive Airway Pressure (CPAP)-Kompaktsystem [12] ähnlich dem Boussignac CPAP-System [13] entwickelt und bislang vor allem zur Therapie postoperativer Hypoxämien und zur Prophylaxe und Behandlung von Atelektasen eingesetzt.

Die Anwendung, die über Mundstück, Maske oder Trachealkanüle möglich ist, bedarf eines Gasflusses (Sauerstoff bzw. Druckluft, 5-15l/min) und eines Manometers zur Adjustierung des vom Flow abhängigen positiven endexspiratorischen Drucks (PEEP). Dieser wird durch einen Coanda-Effekt erzeugt, ähnlich dem Prinzip von Bernouilli, das die Beziehung zwischen der Strömungsgeschwindigkeit von Fluiden bzw. Gasen und deren Druck beschreibt, wobei die Summe derselben konstant bleibt. Bei EzPAP werden die eingesetzten Gase beim Strömen entlang der konvexen Oberfläche des Geräts beschleunigt. Nach Herstellerangabe bewirkt dies einen annähernd 4-fachen Zustrom von Raumluft, wodurch die inspiratorische Sauerstoffkonzentration $\left(\mathrm{FiO}_{2}\right)$ in Abhängigkeit von der Atemtiefe des Patienten relativ konstant bei 35 bis $42 \%$ bleibt [12].

Der Einsatz von Ez-PAP bei COPD-Patienten mit ventilatorischem Versagen erfolgt hierbei nicht zum Zweck einer passiven CPAPTherapie, sondern als optimierte atemtherapeutische Option zu nichtoszillierenden PEP-Ventilen. Ez-PAP leistet der überblähten
Lunge, bei der dadurch eine geringe Compliance vorliegt, Inspirationsunterstützung durch den Coanda-Effekt [12]. Die Exspiration wird gegen den kontinuierlichen Gasfluss ausgeführt und erzielt einen PE(E)P-Effekt [Positive (End) Expiratory Pressure], der dem Bronchialkollaps entgegenwirkt und eine vollständigere Ausatmung bei deutlich verlängerter Exspirationszeit ermöglicht.

Der nachfolgende Beitrag beschreibt eine retrospektive Analyse atemtherapeutischer Maßnahmen mit Ez-PAP, um die Therapieeffekte und Einsatzmöglichkeiten bei Patienten mit schwerer COPD und Lungenemphysem zu analysieren.

\section{Patienten und Methode \\ $\nabla$}

\section{Patienten}

In die retrospektive Analyse wurden 30 Patienten mit COPD Grad D nach GOLD 2013 bzw. lungenfunktioneller Obstruktion im Schweregrad IV nach GOLD 2007 eingeschlossen. Die Patienten befanden sich im Zeitraum von Juli 2011 bis Dezember 2011 stationär auf der pneumologischen Wach- und Weaningstation der Thoraxklinik, Universitätsklinikum Heidelberg. Es handelte sich um 20 Männer und 10 Frauen im medianen Alter von 61,5 Jahren.

Im Median fand sich in der Spirometrie bei 24 der 30 Patienten eine mediane Vitalkapazität (VC) von 1,51 (43,5\%), eine mediane Einsekundenkapazität $\left(\mathrm{FEV}_{1}\right)$ von $0,571(20,8 \%)$ und ein medianer Tiffeneau-Index (Verhältnis VC/FEV $_{1}$ ) von 42,2\%. Bei 21 der Patienten lagen bodyplethysmografische Daten vor, die im Median ein Residualvolumen (RV) von 6,741 (313,5\%), eine totale Lungenkapazität (TLC) von 8,61 (148,3\%) und ein Verhältnis RV zur TLC von $80,6 \%$ aufwiesen. Von 6 Patienten lagen keine spirometrischen und bei 9 keine bodyplethysmografischen Daten vor. Die Bodyplethysmografie wurde nicht durchgeführt aufgrund von Immobilität im Weaning $(n=2)$, starker Dyspnoe $(n=3)$, Versterbens der Patienten im Verlauf $(n=3)$, anderen Ursachen $(n=1)$. Die Raucheranamnese zeigte Ex-Raucher $(n=24)$, aktive Raucher $(\mathrm{n}=5)$ mit einem gesamten medianen Nikotinabusus von 57,8 packyears, und 1 Nieraucher.

Alle Patienten wurden nichtinvasiv $(n=28)$ oder invasiv $(n=2)$ beatmet. Indikationen für die Beatmungstherapie waren chronisches ventilatorisches Versagen im Weaning $(n=5)$, die Einleitung einer nichtinvasiven Beatmungstherapie (NIV) bei akutem ventilatorischen Versagen ( $n=8)$, die Evaluation weiterer Therapieoptionen bei schwerem Lungenemphysem $(n=2)$, akute COPD-Exazerbationen bei vorbestehender NIV $(n=6)$, und Beatmungskontrollen unter NIV $(n=9)(\checkmark$ Tab. 1$)$. Alle Patienten befanden sich unter Langzeitsauerstofftherapie (LTOT) mit einem medianen Fluss von 2,51/min (Intervall 1-61/min). Die medikamentöse Therapie umfasste eine inhalative antiobstruktive Therapie (langwirksame Betamimetika und Muscarinantagonisten) bei allen Patienten $(n=30)$ mit zusätzlichen inhalativen Steroiden $(\mathrm{n}=25)$. Opiate kamen zur Coupierung der Dyspnoe bei 17 Patienten bedarfsorientiert, sowie dauerhaft bei 13 Patienten, eine Therapie mit systemischen Glucocortikoiden bei 13 Patienten zum Einsatz ( Tab.2). Indikation zur Ez-PAP-Therapie war die Optimierung einer nichtoszillierenden intermittierenden PEPTherapie mit Inspirationshilfe zur Behandlung der dynamischen Überblähung im beatmungsfreien Intervall. Die Patienten wurden von einer Atmungstherapeutin individuell betreut. 


\begin{tabular}{|c|c|}
\hline \multicolumn{2}{|l|}{ Patientencharakteristika } \\
\hline Diagnose: COPD D/COPD IV ${ }^{\circ}$ & $\mathrm{n}=30$ \\
\hline medianes Alter (Intervall) & 61,5 Jahre $(40-75)$ \\
\hline Geschlecht & $66 \%$ männlich, $33 \%$ weiblich \\
\hline \multicolumn{2}{|l|}{$\begin{array}{l}\text { Lungenfunktion: } \\
\text { Spirometrie }(n=24) \text {, Bodyplethysmografie }(n=21) \text { keine Daten }(n=6)\end{array}$} \\
\hline Spirometrie & $n=24$ \\
\hline mediane Vitalkapazität [VC] & 1,5I (Intervall: $0,6-3,5)$ \\
\hline \%Soll & $43,5 \%$ (Intervall: $18,2-84$ ) \\
\hline mediane Einsekundenkapazität [FEV1] & 0,57 I (Intervall: $0,35-0,82$ ) \\
\hline \%Soll & $20,8 \%$ (Intervall: $12,9-29,2$ ) \\
\hline mediane VC/FEV1 & $42,2 \%$ (Intervall: $16,3-56,7$ ) \\
\hline Bodyplethysmografie & $\mathrm{n}=21$ \\
\hline medianes Residualvolumen & $6,74$ I (Intervall: $4,53-8,9)$ \\
\hline \%Soll & $313,5 \%$ (Intervall: $246,9-389$ ) \\
\hline mediane totale Lungenkapazität & 8,6I (Intervall: 6,47-10,6) \\
\hline \% Soll & 148,3\% (Intervall: $114-175,3$ ) \\
\hline Verhältnis Residualvolumen zu totaler Lungenkapazität [RV/TLC] & $80,6 \%$ (Intervall: $70,1-89)$ \\
\hline Raucheranamnese & $\mathrm{n}=\mathbf{3 0}$ \\
\hline Nieraucher & $n=1$ \\
\hline Ex-Raucher & $\mathrm{n}=24$ \\
\hline aktive Raucher & $\mathrm{n}=5$ \\
\hline mediane pack years & 57,8 Jahre $(n=18)$ \\
\hline Indikation zur stationären Aufnahme & $\mathbf{n}=\mathbf{3 0}$ \\
\hline Weaning & $\mathrm{n}=5$ \\
\hline akute Einleitung einer nichtinvasiven Beatmung (NIV) & $\mathrm{n}=8$ \\
\hline $\begin{array}{l}\text { Einleitung einer NIV bei chronischer ventilatorischer Insuffizienz, } \\
\text { Evaluation weiterer Therapieoptionen }\end{array}$ & $n=2$ \\
\hline akute COPD Exazerbation/Pneumonie bei bestehender NIV & $n=6$ \\
\hline Beatmungskontrolle unter NIV & $n=9$ \\
\hline
\end{tabular}

Tab. 1 Patientencharakteristika.

\begin{tabular}{|c|c|}
\hline COPD-Therapie & $\mathbf{n}$ \\
\hline nichtmedikamentöse Therapie & 30 \\
\hline Beatmung & 30 \\
\hline nicht-invasiv & $28(93,3 \%)$ \\
\hline invasiv & $2(6,6 \%)$ \\
\hline $\begin{array}{l}\text { Langzeit-Sauerstofftherapie (LTOT) } \\
\text { medianer Fluss 2,5I (Intervall: } 1-6 \text { ) }\end{array}$ & 30 \\
\hline Physiotherapie $^{1}$ & 21 \\
\hline Atemtherapie mit Ez-PAP & 30 \\
\hline medikamentöse Therapie & 30 \\
\hline antiobstruktive/antientzündliche Therapie & 30 \\
\hline handheld devices & 26 \\
\hline LAMA $^{2}$ & 25 \\
\hline $\mathrm{LABA}^{3}$ & 26 \\
\hline $\mathrm{ICS}^{4}$ & 25 \\
\hline $\mathrm{SABA}^{5}$ & 2 \\
\hline SABA/SAMA ${ }^{6}$ & 14 \\
\hline Feuchtinhalation & 14 \\
\hline Sole-Inhalation $(0,9-6 \% \mathrm{NaCl})$ & 14 \\
\hline SABA/SAMA & 6 \\
\hline ICS & 1 \\
\hline \multicolumn{2}{|l|}{ systemische Therapie } \\
\hline Theophyllin & 1 \\
\hline Roflumilast & 1 \\
\hline Kortikosteroide (Prednisolon 2,5 - $15 \mathrm{mg}$ ) & 13 \\
\hline Opiate (Morphinsulfat, Fentanyl) intermittierend & 18 \\
\hline Opiate (Morphinsulfat, Fentanyl) dauerhaft & 13 \\
\hline \multicolumn{2}{|c|}{$\begin{array}{l}1 \text { Physiotherapie: Mobilisation, Gerätetraining, Atemtherapie mit/ohne Hilfsmittel } \\
2 \text { LAMA= long acting muscarinic antagonist (Anticholinergikum, langwirksam) } \\
3 \text { LABA= long acting beta agonist (Beta-2-Sympathomimetikum, langwirksam) } \\
{ }^{4} \text { ICS= inhaled corticosteroids (inhalative Steroide) } \\
{ }^{5} \text { SABA= short acting beta agonist (Beta-2-Sympathomimetikum, kurzwirksam) } \\
{ }^{6} \text { SABA/SAMA = short acting beta agonist (Beta-2-Sympathomimetikum, kurzwirksam)/short acting muscarinic antagonist } \\
\text { (Anticholinergikum, kurzwirksam) }\end{array}$} \\
\hline
\end{tabular}

Tab.2 Medikamentöse und nichtmedikamentöse Therapie. 


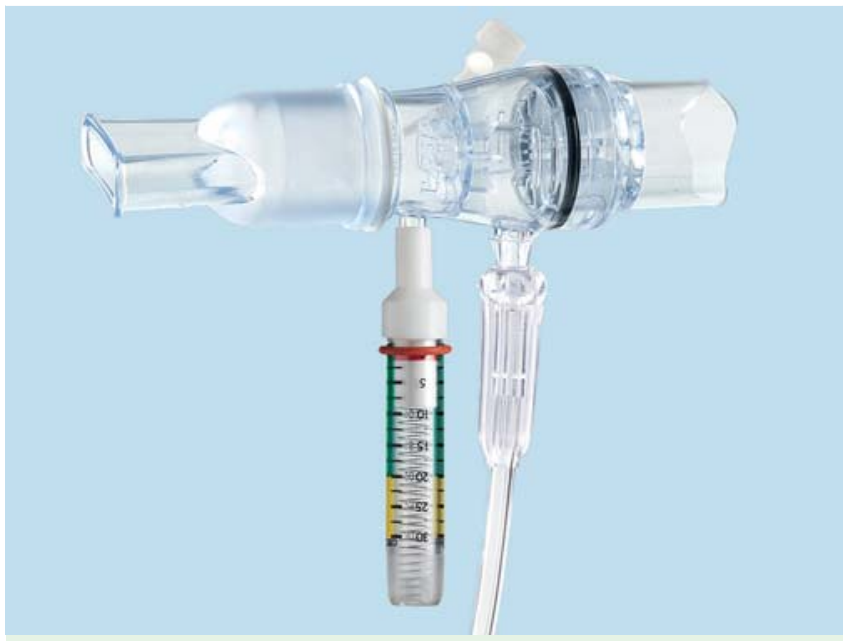

Abb.1 Ez-PAP (Fa. Smiths Medical, Grasbrunn, Deutschland), Gerät mit Mundstück, Anschluss für Gaszufuhr (Luft/Sauerstoff), Anschluss für Messung des Atemwegsdrucks (optional).

\section{Methode}

\section{Ez-PAP}

Die Patienten wurden darin geschult, in den beatmungsfreien Zeiten für 3-5 Minuten tief über Ez-PAP ${ }^{\circledR}$, (Smiths Medical) $\left(\bullet\right.$ Abb.1) bei einem Gasfluss von $6-81 \mathrm{O}_{2}$ ein- und so lange wie möglich konstant auszuatmen. Die Messung der in- und exspiratorischen Druckverhältnisse erfolgte mit einem Druckmessgerät (Kolbenmanometer). Hierbei wurde individuell eine maximale Exspirationszeit etabliert unter Vermeidung eines bronchialen Kollapses. Die Methode wurde nicht-invasiv über das Mundstück $(\mathrm{n}=28)(\boldsymbol{A} \mathbf{A b} \mathbf{b} . \mathbf{2} \mathbf{a})$ und/oder eine unfixierte Maske $(n=8)(\bullet$ Abb. 2b b), sowie auch invasiv mit Filter versehen (Barr-vent-S ${ }^{\circledR}$, Medisize) über eine geblockte Trachealkanüle angewandt $(\mathrm{n}=2)(\bullet$ Abb.3).

Die Exspiration wurde nach Ersteinweisung in die Therapie und in Phasen erhöhter Dyspnoe, z.B. nach körperlicher Belastung oder direkt nach Beatmungsende, atemphysiotherapeutisch manuell - dorsokaudal am Thorax und/oder dorsokranial am Abdomen - unterstützt [14]. Die Patienten führten die Therapie nach umfassender Schulung selbstständig und teilweise mit Unterstützung von Angehörigen durch.

Bei 16 Patienten ohne oder nur mit sehr kurzen Beatmungspausen von einigen Minuten wurde die Therapie wie folgt vorbereitet und durchgeführt:

- 3 Hub Berodual über Spacer

- $5 \mathrm{mg}$ Morphin subkutan

- Einstellung der eingestellten Backup Frequenz auf 0-8 Atemzüge/min

- Erhöhung des Inspirationsdrucks um 3 mbar und Anleitung zur Lippenbremse unter Fullface-Maske oder gezielt langer Ausatmung über die Trachealkanüle

- Präoxygenierung mit 101 Sauerstoff über 3 Minuten

- Beatmungsstopp

- Additive Behandlung mit Ez-PAP sofort im Anschluss über Maske, Mundstück oder Trachealkanüle

- Fortführung der Beatmung bei Hypoxie und Sauerstoffsättigung $\left(\mathrm{SO}_{2}\right) \leq 83 \%$ (pulsoxymetrisch gemessen)

Die Anwendungen fanden unter Monitoring von EKG, Sauerstoffsättigung und nichtinvasiver Blutdruckmessung statt.

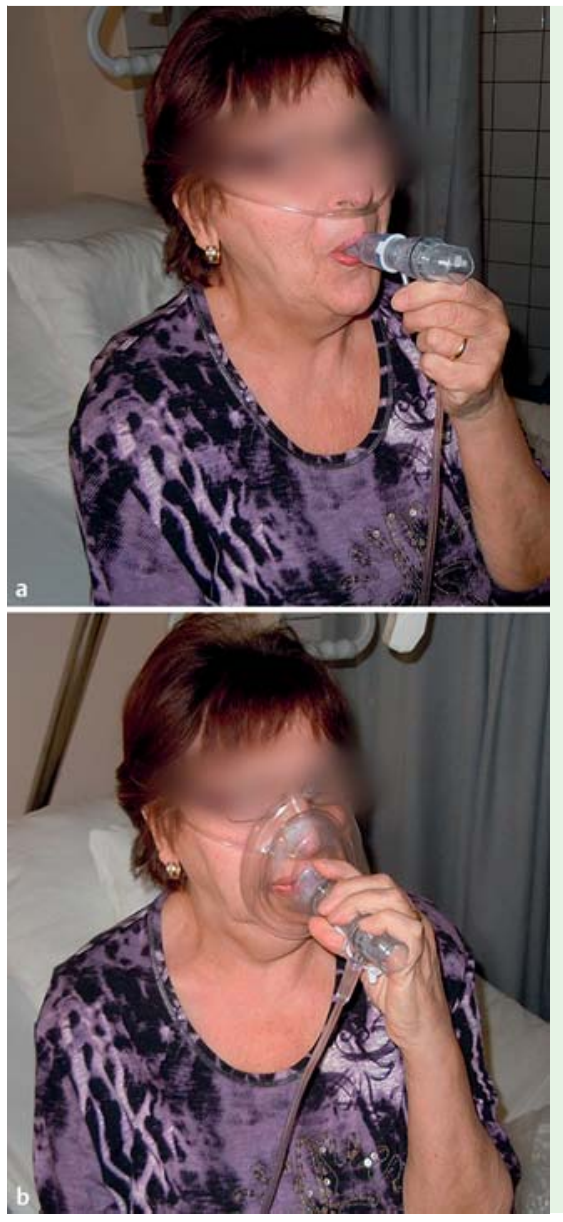

Abb.2 a Therapie mit Ez-PAP über Mundstück. b PEP-Husten mit Ez-PAP und Maske zur tracheobronchialen Stabilisation.

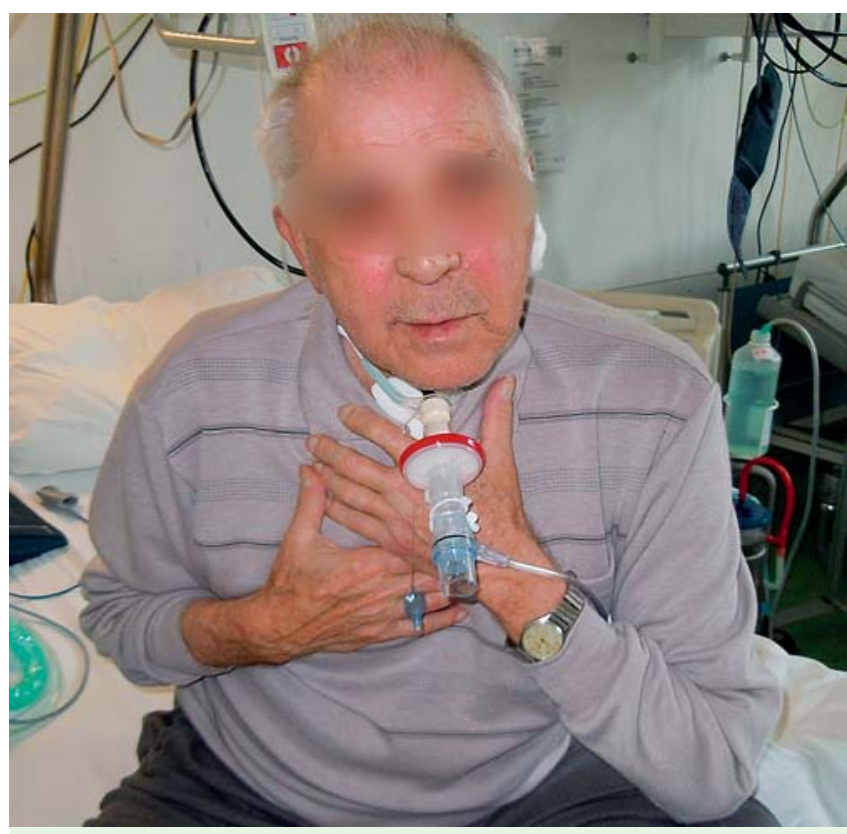

Abb. 3 Selbständige Therapie mit Ez-PAP über Trachealkanüle zu Entblähung und Sekretmanagement. 


\section{Bestimmung von Outcome-Parametern}

30 COPD Patienten erhielten eine Atemtherapie mit Ez-PAP. Aufgrund von Beatmungsabhängigkeit und geringer Belastbarkeit konnten in diesem retrospektiven Ansatz nicht bei allen Patienten alle Outcome-Parameter bestimmt werden.

Die erhobenen Parameter unter Ez-PAP sind mit Angabe der jeweiligen Patientenzahl ausführlich in $\bullet$ Tab. 3 dargestellt:

Tab. 3 Bestimmung von Outcome-Parametern.

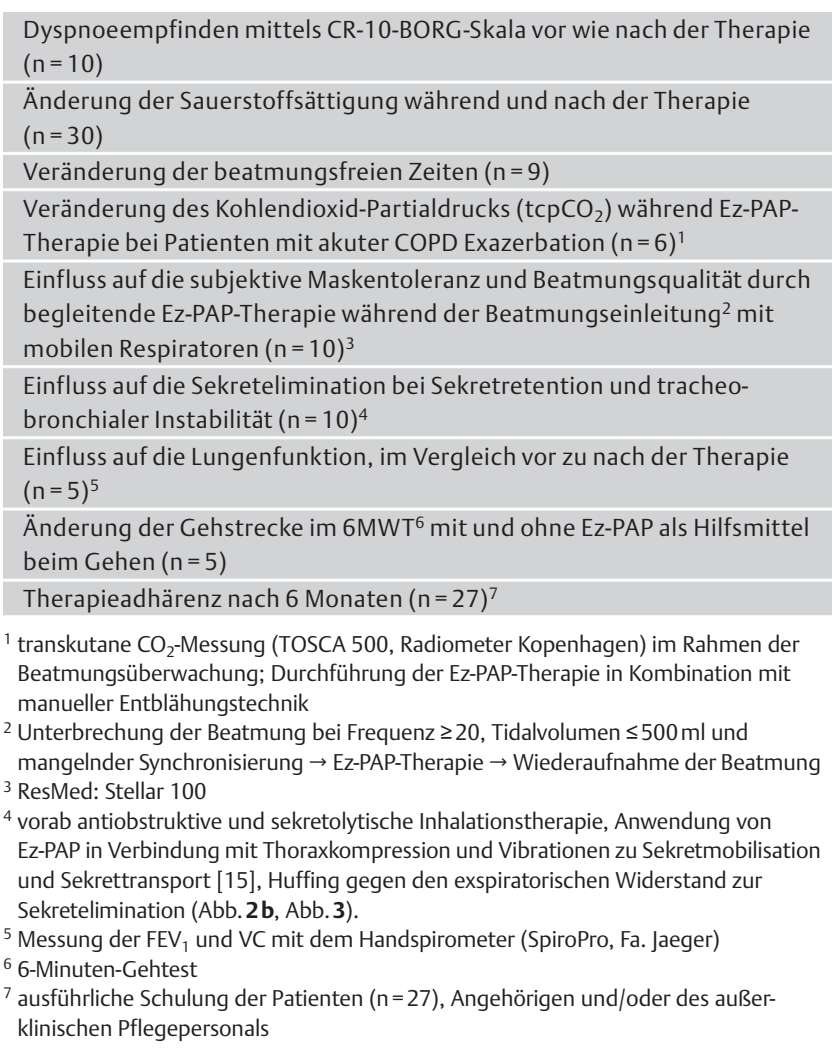

\section{Ergebnisse}

Ez-PAP-CPAP Kompakt-Gerät: Anwendung und

Druckverhältnisse

Es entstanden bei der kurzzeitigen, aktiven Atemtherapie mit Ez-PAP keine kontinuierlichen positiven Druckverhältnisse in der In- und Exspiration. Druckmessungen mit dem Manometer zeigten bei der hier beschriebenen Art der Anwendung, dass bei tiefer Einatmung am Anfang der Inspiration noch negative gegen Ende der Inspiration geringfügig positive Drücke von 1 bis 2 mbar entstehen und sich in der Exspiration ein konstanter positiver endexspiratorischer Druck von 8 bis $10 \mathrm{mbar}$, abhängig von Gasfluss und Exspirationskraft des Patienten und maximal 18 mbar unter therapeutischer manueller Exspirationsunterstützung ausbildet. Es ergaben sich bei einer Anwendungszeit von im Median 3-5 (maximal 12) Minuten Atemzeitverhältnisse von 1:8 bis 1:20. Negative hämodynamische Nebenwirkungen wurden bei keinem Patienten beobachtet $(n=30)$.

\section{Dyspnoeempfinden}

Patienten ( $n=10)$ gaben unmittelbar nach 3-5-minütiger Ez-PAP Therapie eine Besserung der Atemnot im Median um 3,3 Punkte auf der CR-10-BORG-Skala an. Die Besserung hielt 12 bis $60 \mathrm{Mi}-$ nuten an. Patienten mit einem Ruhedyspnoe-Ausgangswert von
3-4 auf der Visuellen Analog-Skala (VAS) konnten nach dem Atmen über das Gerät subjektiv tiefer durchatmen und gaben keine Atemnot mehr an (VAS 0) $(n=2)$. Die sehr schwere bis maximale Atemnot von 6-10 auf der CR-10-BORG-Skala bei 8 Patienten direkt nach Pausierung der Beatmung $(n=8)$ oder nach körperlicher Belastung der Patienten $(n=8)$ wurde gleichermaßen im Median um 3,25 Punkte reduziert (Intervall 2 bis 4 Punkte).

\section{Änderung der Sauerstoffsättigung}

Die Therapie mit Ez-PAP verbesserte die Oxygenierung im Vergleich zur Spontanatmung bei 25 von 30 Patienten während der Anwendung. Eine direkt nach Pausierung der nicht-invasiven Beatmung mittels Herz-Kreislauf-Monitorings beobachtete Hypoxie bis $<70 \%$ Sauerstoffsättigung $\left(\mathrm{sO}_{2}\right)$, die auch bei erhöhter Sauerstoffgabe von $101 / \mathrm{min}$ über eine $\mathrm{O}_{2}$-Maske auftrat $(n=16)$, fiel nach der Behandlung mit Ez-PAP geringer aus, im Median blieb der $\mathrm{SO}_{2} \geq 88 \%$.

\section{Veränderung der beatmungsfreien Zeiten}

Bei 8 Patienten unter langjähriger NIV und aktuell krisenbedingter dauerhafter Beatmung sowie bei 1 Patienten im diskontinuierlichen Weaning wurden wieder beatmungsfreie Zeiten bis zu 6 Stunden (h) (Intervall 2,5 bis $6 \mathrm{~h}$ ) möglich.

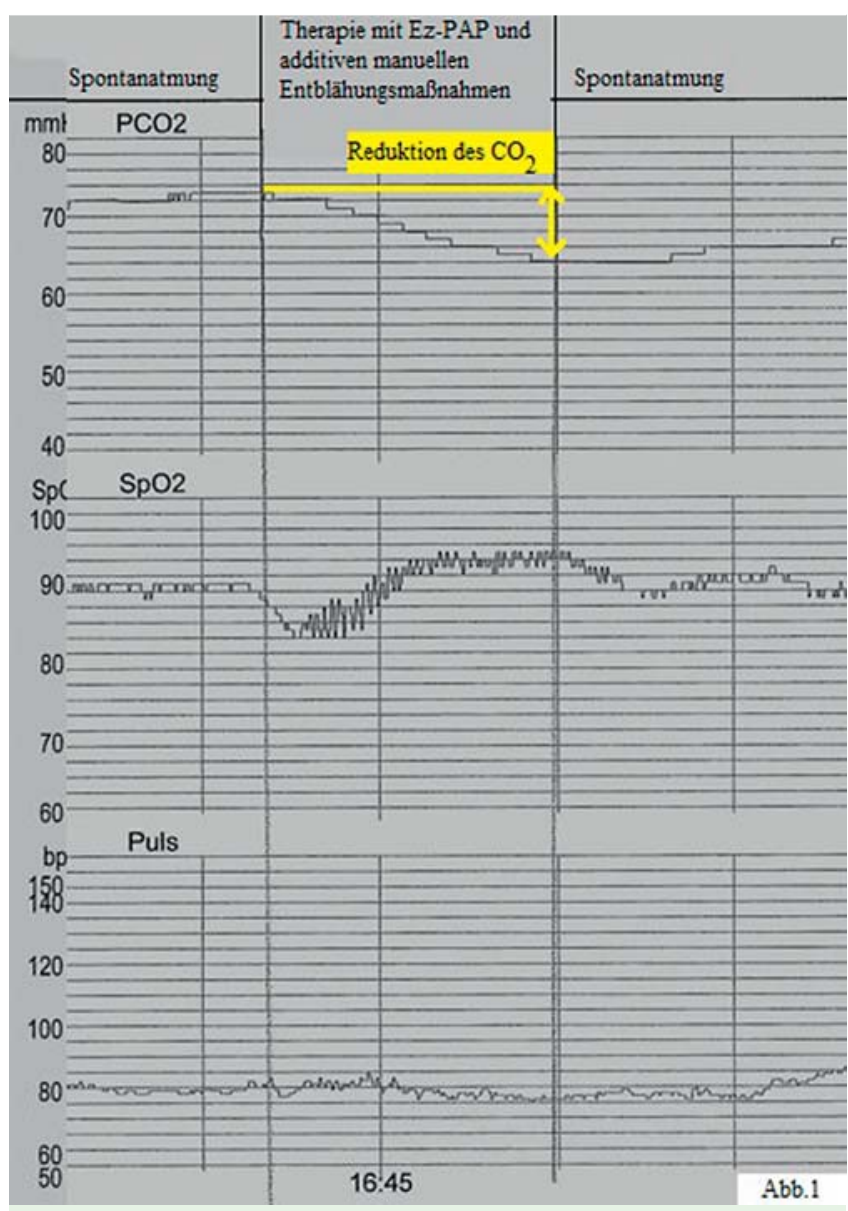

Abb.4 Transkutane $\mathrm{CO}_{2}$-Messung (TOSCA 500, Radiometer Kopenhagen) bei einem Patienten mit akut infektexazerbierter schwerer COPD. Im Verlauf von wenigen Minuten zeigt sich unter Ez-PAP Therapie, kombiniert mit manueller Entblähungstechnik, eine Reduktion des $\mathrm{CO}_{2}$ um $9 \mathrm{mmHg}$ $(73 \rightarrow 64 \mathrm{mmHg})$. 


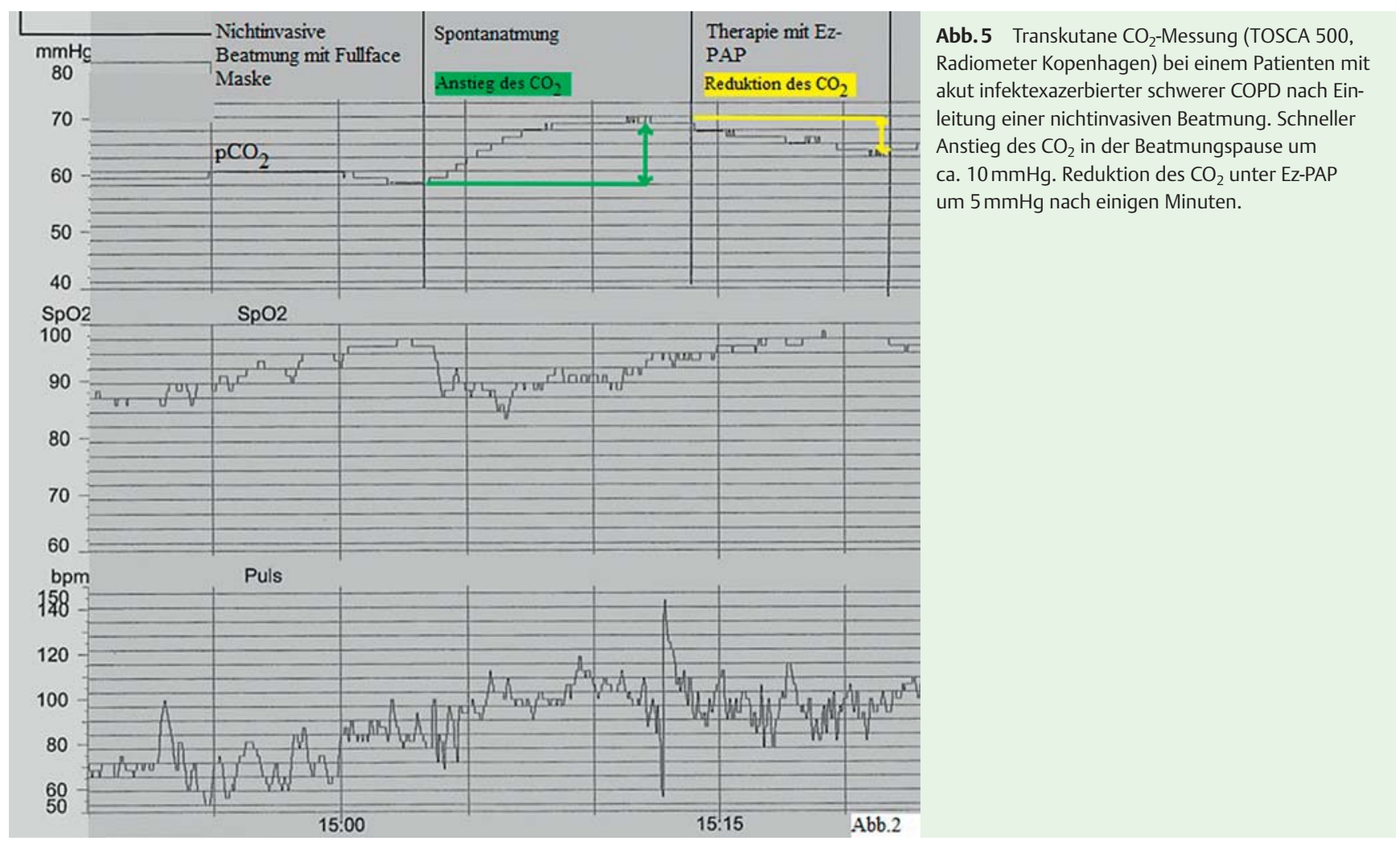

Veränderung des Kohlendioxid-Partialdrucks (tcpCO Im Rahmen der Beatmungsüberwachung bei AECOPD fanden transkutane $\mathrm{CO}_{2}$-Messungen bei 6 Patienten statt.

Während der Atemtherapie mit Ez-PAP in Kombination mit manueller Entblähungstechnik wurde ein Rückgang der Hyperkapnie von initial im Median 75,5 mmHg (Intervall 56 bis $94 \mathrm{mmHg}$ ) um $7,3 \mathrm{mmHg}$ (Intervall -5 bis $-10 \mathrm{mmHg}$ ), ( $\mathrm{n}=6)$ innerhalb von 5 bis 12 Minuten erfasst $(\bullet$ Abb. 4 u. $\odot$ Abb.5).

Einfluss auf Maskentoleranz und Beatmungsqualität nach NIV-Einleitung unter begleitender Ez-PAP-Therapie

Die Methode, intermittierend die Beatmung für Ez-PAP-Therapie zu unterbrechen, führte zu subjektiv ausgezeichneter Maskentoleranz bei den Patienten $(n=10)$, mit guter Synchronisation der Beatmung bei niedrigen Atemfrequenzen $(10-16 / \mathrm{min})$, hohen Atemzugvolumina ( 600 bis $1100 \mathrm{ml}$ ) und moderaten inspiratorischen Beatmungsdrücken (Spitzendruck: 17-28 mbar) sowie PEEP (4-6mbar). Sekretclearance war in der Beatmungspause möglich. Zudem war die Gabe von Opiaten unter NIV während der Ez-PAP-Anwendung seltener notwendig.

\section{Sekretmanagement}

Die Sekretelimination verbesserte sich subjektiv bei allen Patienten mit bestehender Sekretproblematik durch leichteres Abhusten des meist zähen Sekrets $(n=10)$. Bei den invasiv beatmeten Patienten $(n=2)$ konnte durch die Behandlung das Sekret zentralisiert und endotracheal abgesaugt werden. Bei 2 nicht-invasiv beatmeten Patienten wurde die Eröffnung von Atelektasen nach initialer Ez-PAP-Therapie über p.a. Röntgen-Thoraxaufnahme $(\circlearrowleft$ Abb.6) dokumentiert.

\section{Einfluss auf die Lungenfunktion}

Spirometrische Messungen vor und nach 3-minütiger Therapie mit Ez-PAP zeigten eine gesteigerte VC im Mittelwert um $544 \mathrm{ml}$ (Intervall von $+310 \mathrm{bis}+670 \mathrm{ml}, \mathrm{n}=5$ ).

Die $\mathrm{FEV}_{1}$ änderte sich nicht relevant. Der Mittelwert der $\mathrm{FEV}_{1}$ betrug vor Therapie $626 \mathrm{ml}$, nach Therapie $612 \mathrm{ml}$.

\section{6-Minuten-Gehtest (6MWT)}

Bei allen Patienten $(n=5)$, die einen 6MWT durchführten, wurde eine Erweiterung der Gehstrecke im Median um 50,4 m (Intervall von 5 bis $132 \mathrm{~m}$ ) mit Ez-PAP als Hilfsmittel dokumentiert. Im Einzelnen zeigte sich, dass 2 Patienten, die initial nicht in der Lage waren, den 6 MWT durchzuführen (10 und $15 \mathrm{~m}$ ), mit Ez-PAP 45 bzw. $80 \mathrm{~m}$ Wegstrecke erreichten. 2 Patienten mit einer Gehstrecke von initial 210 und $270 \mathrm{~m}$ erweiterten die Gehstrecke auf 225 bzw. $275 \mathrm{~m}$. 1 Patient verbesserte sich von initial 160 auf $292 \mathrm{~m}$.

\section{Therapieadhärenz}

Im Verlauf nach 6 Monaten poststationärer Ez-PAP-Therapie war die Compliance der Patienten gut. 27 von 30 Patienten wurde das Gerät als ambulantes Hilfsmittel rezeptiert, 3 Patienten waren während des Klinikaufenthalts verstorben. 16 der 27 Patienten (59,2\%) gaben eine Nutzung des Geräts von mehrmals täglich bis hin zu 3mal/Woche in Ruhe und unter Belastung an, in Abhängigkeit von der aktuellen Atemnotsituation.

Von diesen 16 Patienten nutzten 11 Patienten Ez-PAP vorwiegend zur Überbrückung der Dyspnoe nach der Beatmungstherapie, aber auch zum Teil für Aktivitäten des täglichen Lebens, wie z.B. Treppensteigen, Körperpflege, Essen $(n=5)$ und führten die Behandlung vorwiegend selbständig und mit Hilfe der Angehörigen durch. ( $\odot$ Tab. 4) 

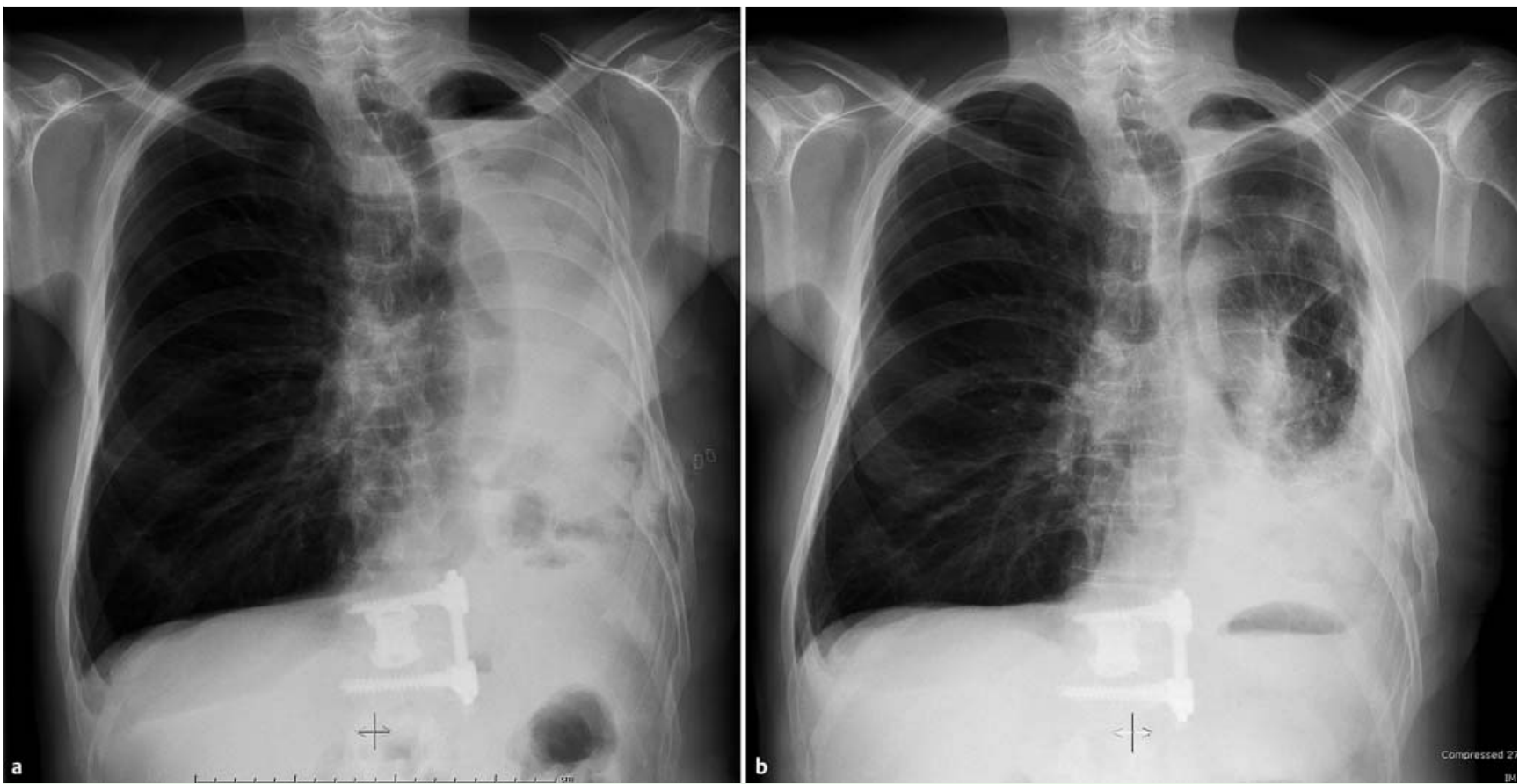

Abb.6 Atelektasenwiedereröffnung mit Ez-PAP bei einem Patienten mit COPD D und Lungenemphysem, bei dem im Vorfeld endobronchiale Ventile eingelegt wurden, als Komplikation ein persistierender Pneumothorax auftrat und infolge dessen eine Oberlappenektomie und Keilresektion des Segments 6 links durchgeführt wurde. a Atelektase li. b Wiedereröffnung der Atelektase li. (Röntgenbilder mit freundlicher Genehmigung von Prof. Dr. C. P. Heußel).

Tab. 4 Ergebnistabelle.

\begin{tabular}{|c|c|c|}
\hline Outcome-Parameter & $\mathbf{n}$ & \\
\hline Atmungstherapie mit Ez-PAP & $\mathrm{n}=30$ & \\
\hline verstorben während des stationären Aufenthalts & $\mathrm{n}=3$ & \\
\hline Schulung von Patienten/Angehörigen/Pflegepersonen & $\mathrm{n}=27$ & \\
\hline entlassungsrelevante Verordnung von Ez-PAP & $\mathrm{n}=27$ & \\
\hline Therapieadhärenz nach 6 Monaten & $\mathrm{n}=16$ & \\
\hline \multicolumn{3}{|l|}{ Ergebnisse der Therapie mit Ez-PAP } \\
\hline Dyspnoeempfinden (CR-10-Borg-Skala) & $\mathrm{n}=10$ & $-3,3$ Punkte (Intervall: $2-4$ ) \\
\hline Zugewinn an beatmungsfreier Zeit & $n=9$ & $+4 \mathrm{~h}$ (Intervall: $+2,5$ bis +6$)$ \\
\hline transkutane $\mathrm{CO}_{2}$-Messung bei akuter COPD- Exazerbation & $n=6$ & $-7,3 \mathrm{mmHg}$ (Intervall: -5 bis -10$)$ \\
\hline Änderung der Vitalkapazität (VC) & $n=5$ & + $544 \mathrm{ml}$ (Intervall: +310 bis +670$)$ \\
\hline Änderung der Einsekundenkapazität (FEV $\left.{ }_{1}\right)$ & $n=5$ & $-14 \mathrm{ml}$ (Intervall: + 15 bis - 36) \\
\hline Änderung der Gehstrecke im 6-Minuten Gehtest (6MWT) & $n=5$ & $+50,4 \mathrm{~m}$ (Intervall: +5 bis +132$)$ \\
\hline
\end{tabular}

\section{Diskussion}

$\nabla$

Im Rahmen unserer retrospektiven Analyse des Stellenwerts einer Flow-PEP- Atemtherapie mit dem CPAP-Kompaktsystem Ez-PAP bei hyperkapnischen Patienten mit schwerer COPD und Lungenemphysem konnten wir zeigen, dass diese einfache Methode die schwere Atemnot lindern kann.

Neben der Beatmungstherapie, Langzeitsauerstofftherapie (LTOT) und leitlinienkonformer medikamentöser Therapie hat sich Ez-PAP hierbei als wichtiger Baustein in unserem Behandlungskonzept der schweren COPD und des Lungenemphysems sowie im Weaning etabliert. Die aktuelle S2k-Leitlinie für prolongiertes Weaning beschreibt die positiven Effekte von CPAP bei obstruktiver Lungenerkrankung im Weaning, z.B. Verringerung der Dyspnoe, Abnahme der Triggerarbeit und Reduktion des iPEEP im Hinblick auf die Spontanatemzeit [16].
Wir nutzten diese Effekte intermittierend, um Tachypnoe mit konsekutiver dynamischer Überblähung und Hypoxie zu vermeiden [2] und den Übergang von der nicht-invasiven oder invasiven kontrollierten BiPAP-Beatmung (Biphasic Positive Airway Pressure) hin zur Phase der Spontanatmung zu überbrücken.

Die hier beschriebene Art der Anwendung ist gut mit den Empfehlungen der Leitlinie der Deutschen Atemwegsliga und der Deutschen Gesellschaft für Pneumologie und Beatmungsmedizin zur Diagnostik und Therapie von Patienten mit chronisch obstruktiver Bronchitis und Lungenemphysem vereinbar [17]. Dort wird die Therapie mit exspiratorischen Stenosen - ohne Oszillation als einfachste Möglichkeit die Lippenbremse, mit Oszillation z.B. Cornet ${ }^{\circledR}$ oder Flutter ${ }^{\circledR}$ - zur nichtmedikamentösen Therapie bezüglich Entblähung, verbesserter Ventilation und Sekretmanagement bei spontan atmenden Patienten angeraten. Dennoch ist die Datenlage zum Stellenwert der PEP-Therapie widersprüchlich. 
Die meisten Studien zur nichtmedikamentösen Behandlung der COPD differenzieren nicht zwischen chronischer obstruktiver Bronchitis und Lungenemphysem und untersuchen hauptsächlich das Sekretmanagement und nicht vorrangig die Behandlung und Vermeidung der dynamischen Überblähung.

So kam die multizentrische randomisierte, kontrollierte Studie von Osadnik et al. zwar zu dem Schluss, dass die Therapie mit PEP-Systemen bei Patienten mit einer akut exazerbierten COPD kurzfristig die Dyspnoe verbessern kann, ihr aber dennoch keine signifikante kurz- oder langfristige Bedeutung in der Behandlung der AECOPD zukommt [18]. Dagegen beschrieben Nicolini et al. [19] in einer aktuellen prospektiven randomisierten Studie die bessere körperliche Leistungsfähigkeit beim Atmen mit PEP-Systemen ( 5 mbar) im 6MWT. Die Probanden erreichten eine weitere Gehstrecke bei besserer Oxygenierung und geringerer Herzfrequenz [19]. Waltersbacher et al. hingegen fanden in einem randomisierten Cross-over-Design keinen Vorteil beim Treppensteigen mit CPAP-Device ( 7 mbar) und zusätzlicher Sauerstoffgabe bezüglich der dynamischen Überblähung und der Atemnot [20]. Die Wirkweise des Ez-PAP ist nicht vollständig geklärt. Die Kombination aus optimierter Inspirationslage mittels des Coanda-Effekts, der den bis zu 4-fachen Zustrom von Raumluft generiert [12], und des flow-bedingten PEP scheint die Atemlast zu senken. Ez-PAP-Therapie führt zu einem bedeutend verlängerten Exspirium und zu einer Reduktion der dynamischen Überblähung, und somit konsekutiv zu geringer Atemfrequenz, höheren Tidalvolumina und geringerer Totraumventilation [2] mit zügigem Abfall des tcpCO $\mathrm{C}_{2}$ während der manuell unterstützten Therapie. Noch gibt es, unseres Wissens nach, keine Studien zur Reduktion von Hyperkapnie unter atmungstherapeutischer PEP- oder CPAP-Behandlung bei COPD und Lungenemphysem.

Den Anstieg der Vitalkapazität nach kurzer Anwendung von EzPAP werten wir als Ausdruck der reduzierten Überblähung, wobei das $\mathrm{FEV}_{1}$ aufgrund der mechanischen, aber nicht bronchodilatatorischen Effekte unverändert bleibt. Inwieweit die maximal erreichbare Entblähung von der Verschiebung des Equal Pressure Point nach proximal durch die Stabilisierung der Bronchien oder aber der Kollateralventilation abhängt, ist fraglich. Hypothetisch kann von Bedeutung sein, ob komplette oder inkomplette Fissuren im Sinne einer kollateralen Ventilation vorliegen.

Möglicherweise sind interlobäre Parenchymbrücken als anatomische Variante [21, 22] für eine besonders wirksame Entlüftung über die kollateralen Atemwege verantwortlich. Weitere Untersuchungen mit exspiratorischen Stenosen unter Beachtung dieses Aspekts sind daher von Interesse.

Obwohl die Linderung der Atemnot nicht dauerhaft ist, kann durch Schulung eine bewusste Atemkontrolle und eine verbesserte Koordination von Atmung und Bewegung erlernt werden. Aus dem subjektiven Gefühl der unmittelbaren Atemerleichterung durch die Anwendung von Ez-PAP bei Atemnot in Ruhe und Belastung sind psychische Entspannungseffekte in Betracht zu ziehen. Wir beobachteten zudem eine gesteigerte Sekretmobilisation, Sekretelimination [23] und die Eröffnung von Atelektasen. Der intermittierende Wechsel zwischen Beatmung und Atemtherapie, die neben der Entblähung auch die Möglichkeit des PEP-Hustens über Maske oder Trachealkanüle bietet, ist offenbar der Clearance förderlich [24].

Ez-PAP lässt sich auch bei beatmungsabhängigen Patienten in kurzen Beatmungspausen einfach anwenden. Die Compliance einer nicht-invasiven Beatmungstherapie kann sich unter begleitender Ez-PAP-Therapie verbessern. Es wird die dynamische Überblähung verringert und dadurch die Beatmungsqualität be- züglich Synchronisation, Atemfrequenz, Atemzugvolumina und benötigtem Beatmungsdruck optimiert. In Phasen hochgradiger dynamischer Überblähung ist Spontanatmung kaum möglich. Mit klassischen Druck- oder Flusstriggern ist eine assistierte Beatmung in solchen Situationen nicht mehr ausreichend möglich [25]. Werden RV, TLC und der iPEEP durch die PEP-Therapie reduziert, verbessert sich die endexspiratorische Position des Zwerchfells, die Zwerchfellbeweglichkeit nimmt zu und die Atmung wird effektiver [26]. Weder die Vermeidung der Hypoxie nach Beatmungsende noch der Zugewinn an beatmungsfreien Zeiten lassen sich retrospektiv präzise darstellen. Theoretisch kann die Ez-PAP-Therapie zu einem gesteigerten intrathorakalen Druck mit hämodynamischen Konsequenzen führen. Allerdings ergeben unsere Daten keinen Anhalt für negative Einflüsse auf die Hämodynamik. Eine kurze Behandlungsdauer von 3 bis 5 Minuten war nötig, um eine optimale Entblähung zu erzielen. Bei der Behandlung mit tiefer Inspiration in Kombination mit assistierter Exspiration entstand, abweichend zu den Angaben des Herstellers, nach unseren Messungen kein kontinuierliches positives Druckniveau wie bei einer konventionellen CPAP-Therapie.

Es ist kritisch anzumerken, dass bei dieser retrospektiven Analyse limitierende Umstände zu berücksichtigen sind. Einerseits handelte es sich um ein kleines, heterogenes Patientenkollektiv, zumeist unter nicht-invasiver, aber auch invasiver Beatmung. Ein Vergleich mit einer Kontrollgruppe fand nicht statt. Allerdings sind die Effekte auf 6MWT, Lungenfunktionstests, Dyspnoe und Reduktion der Hyperkapnie kaum durch einen Placeboeffekt in Analogie zu placebokontrollierten medikamentösen Studien erklärbar. Es gibt keinen Hinweis darauf, dass die kausalen Therapien relevanter Komorbiditäten, wie z. B. die kardiale Dekompensation oder von Pleuraergüssen, Einfluss auf die hier geschilderten Ergebnisse gehabt haben könnten. Andererseits ist die inhalative antiobstruktive Therapie der Patienten während des stationären Aufenthalts optimiert worden und wird somit zu einem gewissen Teil die erzielten Ergebnisse positiv beeinflusst haben [27]. Ein weiterer zu diskutierender Punkt ist, dass in der dargestellten Analyse alle mit Ez-PAP behandelten Patienten eingeschlossen wurden. Neben der Beschreibung der Beobachtungen bezüglich Beatmungsqualität, Beatmungsüberwachung, Beatmungspausen, nahmen nur diejenigen Patienten an zusätzlichen Lungenfunktionstests oder 6MWT teil, die aufgrund der positiven Effekte der Therapie motiviert und körperlich dazu in der Lage waren, sodass sich auch hierdurch eine gewisse Verzerrung ergeben haben kann. Auch erzielten beim 6MWT 2 Patienten bei guter Atemerleichterung aufgrund muskulärer Erschöpfung der Beine lediglich eine geringfügige Erweiterung der Wegstrecke (210 auf $225 \mathrm{~m}, 270$ auf $275 \mathrm{~m}$ ), nachdem der Verlaufs-Gehtest mit Ez-PAP nach kurzer Ruhepause (10 min.) nach dem initialen Gehtest stattgefunden hatte.

Allgemein ist für die Therapie die Fähigkeit, Inspiration und Exspiration über das Gerät zu führen, von Bedeutung. Je nach aktuellem Zustand des Patienten wurde das therapeutische Vorgehen in Bezug auf den verwendeten Flow, Device (Mundstück, Maske, Trachealkanüle) und die manuelle Unterstützung modifiziert. Abschließend gelang die Integration von Ez-PAP nach dem Klinikaufenthalt in den Alltag bei einem überwiegenden Teil der Patienten, abhängig von den kognitiven Fähigkeiten und der Schulung von Angehörigen bzw. Pflegepersonen. Seit Juni 2012 ist diese Therapie aufgrund unserer positiven, hier geschilderten Erfahrungen in unserer Atemtherapie fest etabliert und wird interdisziplinär von Atmungstherapeuten, Pflegepersonal und Physiotherapeuten angewandt. 


\section{Schlussfolgerung}

Ez-PAP scheint mit einfacher, mechanischer Methode die dynamische Überblähung bei der schweren COPD zu reduzieren, die Atmung zu ökonomisieren und die Atempumpe teilweise zu entlasten. Verbessertes Sekretmanagement und die Wiedereröffnung von Atelektasen tragen zum nachhaltigen Therapieerfolg bei. Auch außerklinisch lässt Ez-PAP einen relevanten therapeutischen Nutzen erkennen. Zusammenfassend scheint es sich bei Ez-PAP um eine wirksame Therapie bei Patienten mit schwerer COPD und Lungenemphysem zu handeln. Weitere prospektive Daten sind notwendig, um den endgültigen Stellenwert dieser vielversprechenden Methode besser einordnen zu können.

\section{Interessenkonflikt}

$\nabla$

Gabriele Iberl erhielt von der Fa. Smiths Medical ein Vortragshonorar. Die anderen Autoren geben keinen Interessenkonflikt an.

\section{Literatur}

1 Incalzi RA, Scarlata S, Pennazza G et al. Chronic Obstructive Pulmonary Disease in the elderly. Eur J Intern Med 2014; 25: 320-328

2 Macklem PT. Therapeutic implications of the pathophysiology of COPD. Eur Respir J 2010; 35: 676 - 680

3 Tantucci C. Expiratory flow limitation definition, mechanisms, methods, and significance. Pulm Med 2013: 749860

4 Sciurba FC, Ernst A, Herth FJ et al. VENT Study Research Group. A randomized study of endobronchial valves for advanced emphysema. $\mathrm{N}$ Engl J Med 2010; 363: 1233-1244

5 Osthoff M, Jenkins C, Leuppi J. Chronic obstructive pulmonary disease a treatable disease. Swiss Med Wkly 2013; 143: w 13777

6 Nowobilski $R$, Włoch $T$, Płaszewski $M$ et al. Efficacy of physical therapy methods in airway clearance in patients with chronic obstructive pulmonary disease: a critical review. Pol Arch Med Wewn 2010; 120: $468-477$

7 Andrews J, Sathe NA, Krishnaswami S et al. Nonpharmacologic airway clearance techniques in hospitalized patients: a systematic review. Respir Care 2013; 58: 2160-2186

8 Perez Bogerd S, Selleron B, Hotton $R$ et al. Chest physiotherapy techniques - can they reduce hyperinflation. Rev Mal Respir 2009; 26: $1107-1117$

9 Martin AD, Davenport PW. Extrinsic Threshold PEEP Reduces Postexercise Dyspnea in COPD Patients: A Placebo-controlled, Doubleblind Cross-over Study. Cardiopulm Phys Ther J 2011; 22: 5-10

10 Bubulij C. Atmungstherapeutische Maßnahmen zur Sekretolyse und Sekretentfernung. In: Schwabbauer N, Riessen R (Hrsg). Sekretmanagement in der Beatmungsmedizin. Bremen: UNI-MED; 2010: 44-49
11 Elkins MR, Jones A, van der Schans C. Positive expiratory pressure physiotherapy for airway clearance in people with cystic fibrosis. Cochrane Database Syst Rev; 2006: CD003147

12 Rieg $A D$, Stoppe $C$, Rossaint $R$ et al. EzPAP zur Therapie der postoperativen Hypoxämie im Aufwachraum. Der Anaesthesist 2012; 61: 867874

13 Sehlin M, Törnell SS, Öhberg $F$ et al. Pneumatic performance of the Boussignac CPAP system in healthy humans. Respir Care 2011; 56: $818-826$

14 van Gestel A, Teschler H (Hrsg). Physiotherapie bei chronischen Atemwegs- und Lungen-Erkrankungen. Berlin: Springer-Verlag; 2010

15 Weise S, Kardos P, Pfeiffer-Kascha D et al. Empfehlungen zur physiotherapeutischen Atemtherapie. München-Orlando: Dustri-Verlag; 2008: $4-5$

16 Schönhofer B, Geiseler J, Dellweg D et al. Prolongiertes Weaning. Pneumologie 2014; 68: 19-75

17 Vogelmeier C, Buhl R, Criée CP et al. Guidelines for the diagnosis and therapy of COPD issued by Deutsche Atemwegsliga und Deutsche Gesellschaft für Pneumologie und Beatmungsmedizin. Pneumologie 2007; 61: e1-40

18 Osadnik CR, McDonald CF, Miller BR et al. The effect of positive expiratory pressure (PEP) therapy on symptoms, quality of life and incidence of re-exacerbation in patients with acute exacerbations of chronic obstructive pulmonary disease: a multicentre, randomised controlled trial. Thorax 2014; 69: 137-143

19 Nicolini A, Merliak F, Barlascini C. Use of positive expiratory pressure during six minute walk test: results in patients with moderate to severe chronic obstructive pulmonary disease. Multidiscip Respir Med 2013; $8: 19$

20 Walterspacher S, Walker DJ, Kabitz HJ et al. The effect of positive airway pressure on stair-climbing performance in severe COPD patients. COPD 2013: $193-199$

21 van Rikxoort EM, Goldin JG, Galperin-Aizenburg $M$ et al. A method for the Automatic quantification of the completeneness of pulmonary fissures: evaluation in a database of subjects with severe emphysema. Eur Radiol 2012; 22: 302-309

22 Koenigkam-Santos $M$, de Paula WD, Owsijewitsch $M$ et al. Incomplete pulmonary fissures evaluated by volumetric thin-section $\mathrm{CT}$ : semi quantitative evaluation for small fissure gaps identification, description at prevalence and severity of fissural defects. Eur J Radiol 2013; 82: $2365-2370$

23 Spinelli E, Timpano S, Fogazzi A et al. 18q deletion in a cystic fibrosis infant, increased morbidity and challenge for correct treatment choices: a case report. Ital J Pediatr 2011; 37: 22

24 Hill K, Patman S, Brooks D. Effect of airway clearance techniques in patients experiencing an acute exacerbation of chronic obstructive pulmonary disease: a systematic review. Chron Respir Dis 2010; 7: 9-17

25 Tobin MJ, Laghi F, Jubran A. Ventilatory failure, ventilator support, and ventilator weaning. Compr Physiol 2012; 2: 2871-2921

26 Weise S, Kardos P, Pfeiffer-Kascha D et al. Empfehlungen zur physiotherapeutischen Atemtherapie. München-Orlando: Dustri-Verlag; 2008: 16

27 Dhand R, Dolovich M, Chips B et al. The role of nebulized therapy in the management of COPD: evidence and recommendations. COPD 2012; 9: $58-72$ 